\title{
IL CINEMA E LA FILOSOFIA: BREVE PASSEGGIATA ALLA RICERCA DI NIETZSCHE
}

Antonio Freddi *

Ho potuto scrivere sul cinema quando dei problemi filosofici mi hanno spinto a cercare delle risposte nel cinema, anche se queste risposte rimettevano sul tappeto altri problemi.

(Gilles Deleuze)

\section{Riassunto}

In questo articolo intendo svolgere una riflessione sul rapporto tra filosofia e cinema. Mi soffermerò in generale sulla capacità del cinema di veicolare in modo peculiare concetti e quindi sulla sua specifica validità epistemologica. In particolare prenderò in considerazione la possibilità di individuare e approfondire le idee di Nietzsche attraverso l'analisi di alcuni film. In appendice accennerò brevemente alle possibilità didattico-filosofiche del cinema.

Parole chiave: Cinema. Concettimmagine. Epistemologia. Nietzsche. Prospettivismo. Didattica.

\begin{abstract}
The main purpose of this article is to reflect upon the relation between philosphy and cinema. The author tries and deepens the capability of cinema to transfers concepts in a peculiar way and its specific epistemological validity. Particularly it is taken into account the possibility to investigate Nietzsche's thought the analysis of certain movies. In the annex two examples of philosophical-educational use of movies are sketched.

Dottore di ricerca $(\mathrm{PhD})$ in filosofia (conseguito presso Università del Piemonte Orientale). Laurea $(\mathrm{Ba}+\mathrm{Ma})$ in Filosofia presso Università degli studi di Parma, Parma, Italia. Università del Piemonte Orientale "A. Avogadro", Vercelli, Italia - Dipartimento di Studi Umanistici. Cultore della materia (Voluntary Fellow) in Storia della filosofia moderna e contemporanea presso Università del Piemonte Orientale "A. Avogadro", Vercelli, Italia. E-mail: antfrd@gmail.com
\end{abstract}


Keywords: Cinema. Image-concepts. Epistemology. Nietzsche. Perspectivism. Education.

\section{Cinema e filosofia: spunti teorici}

\subsection{Perché accostare cinema e filosofia?}

La filosofia si è storicamente sviluppata in forma letteraria e non attraverso immagini; però perché non concepire qualcosa di diverso? Dopotutto nella cultura occidentale la visione ha un ruolo centrale rispetto a tutti gli altri sensi. Inoltre perché appiattirsi sull'idea che il pensiero debba avere un rapporto pressoché unico con il linguaggio discorsivo? Si potrebbe pensare ad un mondo alternativo dove la cultura filosofica viene svolta attraverso immagini, musica o arti corporee come la danza? Forse no, però un modo per saggiare questa possibilità è provare a considerare il cinema una forma di pensiero.

A favore di un tale tentativo si può osservare che la filosofia, negli ultimi due secoli, sembra essersi indirizzata verso orientamenti che possiamo, un po' coraggiosamente, definire cinematografici: a partire dall'esistenzialismo ottocentesco tedesco (Schopenauer e Kierkegaard), successivamente con Nietzsche, con l'esistenzialismo francese e con l'ermeneutica (Heidegger), è stata rimessa in questione la razionalità puramente logica (nel senso di logos) introducendo nel processo di comprensione del reale anche l'elemento affettivo e mostrando l'importanza dell' aspetto esperienziale nell'affrontare i problemi filosofici. Per esprimere le intuizioni di tali filosofi in merito ai limiti di una razionalità esclusivamente logica e in merito agli ambiti del mondo che non sembrano raggiungibili senza l'emozione e la partecipazione, il cinema può offrirci un linguaggio più appropriato rispetto alla parola scritta. Forse esso può superare l'ostacolo che il linguaggio discorsivo oppone al pensiero, per dirla con Nietzsche e con Wittgenstein.

Oltre a fornire una modalità diversa con la quale affrontare i problemi filosofici, il cinema è sorgente di nuove proposte. Per dirla con J. L. Nancy, non è la filosofia che si occupa di cinema ma "[...] è piuttosto il cinema che si occupa di filosofia, ponendo le domande". I film andrebbero considerati

1 NANCY, J. L. L'evidence du film. Abbas Kiarostami, Bruxelles: Yves Gevaert Editeur, 2001. tr. it. Abbas Kiarostami. L'evidenza del film, Donzelli: Roma, 2004. p. 103. 
come forme di pensiero; inoltre rispetto alla filosofia scritta, il cinema riesce a chiamare in causa anche il cuore e la pancia, oltre al cervello. Personalmente i film che trovo maggiormente piacevoli sono quelli in cui mi sembra possibile confrontarmi con ciò che essi dicono intorno alle questioni che ci appassionano o che fanno sorgere il bisogno di porsene altre.

Infine un ulteriore elemento che avvicina cinema e filosofia è, come cercherò di approfondire in seguito, la comune capacità di creare: come ha osservato G. Deleuze, la filosofia crea concetti e il cinema crea blocchi movimento/durata ${ }^{2}$.

\subsection{Quando un film è filosofico?}

Qualsiasi film può offrire qualcosa su cui riflettere, non solo quelli, rari, propriamente filosofici o quelli, meno rari, considerati "intellettuali". Così come i fatti apparentemente più insignificanti della nostra vita possono generare impreviste riflessioni di sconvolgente profondità, la stessa capacità va riconosciuta allo spettacolo di cento minuti di vita concentrata, anche se si tratta di un film commerciale. Come per ogni forma d'arte, anche per il cinema la riflessione può e deve andare ben al di là delle intenzioni e dei pensieri degli autori (tralasciando la questione della esistenza e della natura degli autori che per il cinema è assai complessa).

Non intendo qui soffermarmi sui dubbi espressi a proposito delle finalità del cinema da parte di una forse erronea interpretazione della scuola critica francofortese che vorrebbe il cinema relegato al ruolo di strumento di asservimento dell'individuo da parte della società capitalistica. Neppure sulla colpevole diffidenza nei confronti verso il cinema dovuta ai residui della scuola estetica crociana che mal digerisce la commistione tra arte contemporanea e tecnica che alimenta il cinema. Vorrei però far notare che, almeno in molti paesi il cinema è ancora visto soprattutto come intrattenimento e non è preso, a mio avviso, abbastanza sul serio da parte della filosofia; ma di questo non ci si può troppo lamentare, considerato che dopotutto questo destino è stato condiviso per vari anni con tutta la letteratura.

2 DELEUZE, G. Qu'est-ce que l'acte de création?, conferenza tenuta presso la Fondazione Femis il 17 Marzo 1987, tr. it. Che cos'è l'atto di creazione, Cronopio: Napoli, 2003. p. 10. 


\subsection{L'elemento patico del cinema}

Ho detto che il cinema introduce le emozioni, chiamando in causa la pancia e il cuore: questo però avviene senza scacciare la razionalità bensì ridefinendola. Sembra quindi possibile un accostamento con filosofi come Nietzsche, Kierkegaard e Heidegger, i quali hanno ritenuto che la realtà potesse essere introdotta sensitivamente grazie ad una comprensione allo stesso tempo razionale e affettiva che alcuni definiscono logo-patica. L'introduzione della sensazione deve produrre il raggiungimento di un sapere valido sfruttando un certo tipo di impatto. In questo senso sapere qualcosa non significa semplicemente avere delle informazioni, ma vuol dire soprattutto essersi aperti ad un certo tipo di esperienza ed essersi lasciati prendere da una cosa attraverso l'esperienza viva. Si tratta di un sapere diverso da quello trasmissibile attraverso il testo scritto in quanto risulta non dicibile, non trasmissibile, a chi non abbia condiviso la stessa esperienza.

Tali caratteristiche di vitalità si ritrovano nel linguaggio cinematografico. Infatti un resoconto scritto o verbale di un film, per quanto dettagliato o approfondito, non è assolutamente in grado di sostituirne la visione: l'esperienza viva non può entrare in un resoconto perché si tratta di una componente cognitiva che non può essere veicolata dall'informazione logica sebbene fornisca una dimensione comprensiva del mondo. Ovviamente anche la sola componente patica non è sufficiente: non basta vedere un film per fare filosofia, ma bisogna interagire con le sue componenti logiche, cogliere l'idea o il concetto trasmessi dalle immagini.

\subsection{La peculiare concettualità del cinema}

Julio Cabrera, in Cine: 100 años de filosofia. Una introducciòn a la filosofía a través del análisis de películas ${ }^{3}$, sostiene che il cinema, visto filosoficamente, è la costruzione di "concettimmagine", un tipo di concetto visivo strutturalmente assai diverso dai tradizionali concetti della filosofia scritta, definibili come "concettidea". Tali concettimmagine cercano di pro-

3 CABRERA, J. Cine: 100 años de filosofia. Una introducciòn a la filosofía a través del análisis de películas, Barcelona: Editorial Gedusa, 1999. tr. it. Da Aristotele a Spielberg. Capire la filosofia attraverso i film, Milano, Mondadori, 2000. 
durre nello spettatore un impatto emotivo che allo stesso tempo dica qualcosa sul mondo, cioè che tramite la componente emotiva veicoli un contenuto cognitivo, con valore di verità e universalità sebbene ridefinite all'interno della ragione logopatica. Il tipo di universalità del cinema è, per esempio, della possibilità piuttosto che della necessità: non riguarda necessariamente tutti ma quello che potrebbe succedere a chiunque. I concettimmagine si realizzano non in modo istantaneo come i concetti della filosofia scritta ma nel corso di una durata temporale: o nell'intero sviluppo di un film, o nelle azioni di un particolare personaggio, o in una particolare situazione. Per esempio i film Old Boy e Sympathy for Lady Vengeance di Park Chan-wook possono essere considerati nel loro complesso concettimmagine, sviluppi logopatici della vendetta che si completa con il termine dei film.

Tali concetti non si posizionano però necessariamente sulla superficie delle immagini e degli eventi del film ma anche a livelli più profondi e astratti. Infatti anche in film fantastici, irreali o che comunque non sembrano riguardarci da vicino, si trovano problematiche concernenti l'uomo e i suoi valori, in quanto si presentano eventualità possibili a livello concettuale anche se non empirico. Il linguaggio del cinema è inevitabilmente metaforico, ma la natura fittizia o immaginaria del contenuto di un film non impedisce l'avvicinamento ad una verità; anzi, talvolta, è proprio l'allontanamento dal quotidiano a farci scorgere qualcosa che altrimenti non vedremmo. Inoltre il nascondersi e l'inevitabilità delle metafore e dei concetti fanno si che la bellezza estetica e la rilevanza intellettuale del film non siano garanzia di un contenuto filosofico e problematico: potenziale emotivo illuminante e quindi ottime esperienze filosofiche si possono trovare anche in film di serie B.

In realtà $i$ concettimmagine sono già presenti nella filosofia (per esempio in Heidegger) e soprattutto nella letteratura: il cinema però si distingue per il massiccio impiego che ne fa e per la particolare tecnica con cui li rende peculiari. Infatti, mentre la filosofia scritta e la letteratura offrono gli ingredienti necessari per crearsi personalmente le immagini, il cinema, grazie alla pienezza dell'esperienza visiva che include temporalità e movimento tipici della realtà esperita, amplifica l'impressione di realtà e quindi l'esperienza indispensabile allo sviluppo dei concettimmagine. Attraverso spazialità e temporalità, grazie a montaggio, rimontaggio, inversione, spostamento e particolare taglio delle immagini, offre apertamente ciò che in letteratura è soltanto suggerito. 
Nonostante questa immediatezza il cinema mi sembra offrire soluzioni epistemiche e morali aperte, problematiche e dubbiose ai problemi proposti: la soluzione definitiva non compete al cinema, ancor meno che alla filosofia tradizionale. Esso pone di fronte ad un'esperienza aperta sempre in procinto di riscoprirsi e in perpetua fuga dinanzi ai canoni che cercano di imprigionarla in una casistica ben definita. Mentre la conoscenza scritta pretende spesso di offrire soluzioni definitive e rassicuranti, l'immagine cinematografica non può non essere problematica, destrutturante, distorcente. Il cinema introduce il singolare, il caso la sorpresa, l'errore, la contingenza, il dubbio senza risposta; vive di crisi, di sconcerto e rifugge la logica ordinaria e prevedibile; non conferma mai nulla, anche in film che sembrano celebrare un ordine rassicurante (per esempio i film più esplicitamente schierati e retorici sono, loro malgrado, autoironici). L'immagine, malgrado i desideri degli autori, istilla e concede sempre dubbi prestandosi ad interpretazioni ulteriori: riapre questioni che si vorrebbero chiudere, cerca lo straordinario ovunque, anche dove non ci si aspetterebbe di trovarlo. Mentre la filosofia scritta ha spesso cercato di organizzare, ordinare e giustificare la molteplicità della vita, il cinema preferisce, anche inconsapevolmente, l'incomprensibile e il molteplice. Mentre la filosofia vorrebbe riconciliare l'umanità, il cinema non vi può riuscire: anche l'happy end più sfacciato suona artificioso, si mette in dubbio da solo; non riesce a non problematizzarsi, a prendersi sul serio e come definitivo ${ }^{4}$.

Rispetto alla trattazione filosofica tradizionale il cinema ha un approccio sovversivo: propone il particolare, il sensibile, le molteplici prospettive, l'adesione al vissuto, la trasformazione delle idee in esperienza viva. In esso soggettività e oggettività, universalità e verità, letterale e metaforico si colorano di peculiari note in quanto i suoi concetti penetrano nella cosa stessa tramite l'esperienza.

Perché allora non fare semplicemente filosofia con il cinema e abbandonare la forma scritta? Una prima risposta può essere che non è possibile raggiungere ogni tipo di concetto con le tecniche cinematografiche. Per esempio, già solo rispetto alla letteratura è minore la capacità di rendere i processi psicologici elaborati che avvengono all'interno dei personaggi:

4 Forse sarebbe possibile approfondire un possibile nesso con il concetto di "indecidibile" in Derrida. 
sono, per esempio, falliti i tentativi di portare sullo schermo Proust o Joyce. Inoltre bisogna anche considerare che forse il solo suggerimento mentale (attuato dalla parola scritta), sebbene meno efficace nella penetrazione viscerale, permette in potenza lo sviluppo di una maggiore gamma di concetti.

\subsection{La resa dei concetti nel cinema}

J. Cabrera individua tre principali tecniche specifiche del cinema volte a costruire particolari concettimmagine. La prima è il multiprospettivismo. Il cinema permette di muoversi tra molteplici punti di vista, sia spazialmente con il movimento delle camere e lo spostamento delle locations, sia temporalmente muovendosi nel tempo storico o attraverso brevi flash back, sia psicologicamente passando frequentemente dalla prima alla terza o ad altre persone fino alla profondità dell'introspezione soggettiva (pur con certi limiti). Questo multiprospettivismo può sia soffermare l'attenzione sulla prospetticità di ogni visione, sia sulla possibilità di omniscienza e omniprospetticità che il montaggio, i tagli delle immagini e i movimenti delle camere permettono di accentuare. Per esempio la giustapposizione di un flash back interiore allo svolgimento della scena esterna, offre due punti di vista con un effetto che è assai difficile da realizzare in letteratura. La seconda tecnica è la capacità del cinema di manipolare a piacere tempo e spazio: basta pensare ai film Reservoir Dogs, Pulp Fiction di Quentin Tarantino e Memento di Christopher Nolan. In questo modo il cinema può gestire a piacere le coordinate spazio-temporali, facendo vivere in prima persona allo spettatore il divenire e i cambiamenti la cui sola descrizione scritta rischia di non essere a fondo compresa. La terza ed ultima tecnica è il montaggio, che costituisce la sintassi del film.

\subsection{Validità epistemologica del cinema}

Si è detto che il cinema è costitutivamente fittizio e particolare: può allora ambire a valori universali e veritieri?

In realtà la fantasia, il paradosso e il molteplice che rendono artificioso, non rigido e a volte irreale il cinema sono stati e sono ampiamente sfruttati anche nell'ambito della stessa filosofia scritta nel suo cammino verso l'universalità.. Inoltre il cinema si pone sempre in modo problematicamente costruttivo nel dibattito tra particolare e universale, mostrando come il par- 
ticolare possa condurre all'universale, attraverso un'induzione che definirei empatica. Ogni celebrazione, condanna o rappresentazione non riguarda soltanto l'avvenimento narrato in sé ma anche la sua astrazione (la guerra, l'amore, l'amicizia, la fedeltà, ...). Non solo: attraverso l'emozione, che solo il particolare può suscitare (di solito non ci si emoziona per la guerra in astratto), il concetto colpisce direttamente il cuore degli spettatori: si tratta di una sensibilizzazione concettuale con cui arriva più rapidamente ed efficacemente al cervello, assai prima di quanto farebbe con un intero trattato sulla guerra ed i suoi mali. Si tratta di una "riflessione logopatica di portata universale"s.

Questo per giustificare l'universalità del particolare. Ma per passare dal fittizio alla verità? Innanzitutto si può osservare che l'artificio non è di per sé un limite alla conoscenza: si pensi alle allegorie, ai miti e ai cosiddetti esperimenti mentali di cui è piena la filosofia (in Platone, Descartes, Nietzsche, Searle, Davidson, ecc.). Però il cinema non può convincerci in modo definitivo della verità di quanto afferma: il suo carattere emotivo presenta le idee più che costringere ad accettarle, apre a possibili sensi, ci obbliga a sentire, ad immedesimarci, ad assumere punti di vista. L'emozione interagisce senz'altro con il logos, ma poi servono ulteriori elementi oggettivi per la decisione, che resta nelle mani dello spettatore, in merito alla verità e alla falsità. Quindi per cogliere filosoficamente un film bisogna trattarlo come oggetto concettuale, come concetto visuale in movimento: il cinema è filosofico nel senso che un film può essere analizzato dal punto di vista concettuale. In questo senso l'impatto emotivo non distoglie ma rende coscienti, ci fa sprofondare nella problematicità dolente della realtà più della parola scritta.

Non va infine sottovalutata l'azione didattico-illustrativa del cinema. Opere cinematografiche che trattano direttamente di autori o temi filosofici possono rivelarsi assai utili per avvicinare molte persone a realtà ritenute spesso erroneamente astruse e inavvicinabili. Inoltre, poiché, come spiegato in precedenza, molti concetti espressi dai filosofi possono essere "vissuti" e quindi meglio "compresi" attraverso la visione di un film, anche in ambito scolastico o accademico è a mio giudizio percorribile la via di una triangolazione tra testo scritto, docente e pellicola: sia per stimolare l'interesse, sia

${ }^{5}$ CABRERA, 2000, p. 23. 
per agevolare la comprensione sfruttando la visualizzazione dei concetti, sia per favorire l'approfondimento e l'apprendimento per scoperta personale.

\section{Nietzsche nel cinema}

In questo secondo capitolo, dopo un breve cenno ai caratteri che rendono Nietzsche un filosofo "cinematografico", mi dedico all'analisi di due registi che presentano tratti nietzscheani e soprattutto di alcuni film (affrontati in ordine cronologico) che si prestano ad essere visti attraverso la lente del pensiero di Nietzsche.

\subsection{Caratteri generali del rapporto Nietzsche-cinema}

Alcune caratteristiche del pensiero di Nietzsche sembrano ritrovarsi nel cinema in generale. Innanzitutto il prospettivismo, cioè l'idea che ogni conoscenza sia prospettiva, a mio avviso avvicina enormemente la settima arte al pensiero di Nietzsche. Ho già illustrato in un precedente paragrafo in che senso il cinema sia una realizzazione di multiprospettivismo e non intendo qui dilungarmi sul prospettivismo che Nietzsche propone soprattutto in opere come Al di là del bene e del male, Genealogia della morale, Crepuscolo degli idoli e nei Frammenti postumi (o nella Volontà di Potenza): esso si può condensare nella frase "i fatti non esistono, esistono solo interpretazioni".

Anche la potenziale tendenza del cinema a mostrare l'omniscienza e la omniprospetticità, attraverso il montaggio, i tagli delle immagini e i movimenti delle camere, come proposta di sapere assoluto si accosta ad alcune recenti, anche se discutibili, interpretazioni del prospettivismo di Nietzsche ${ }^{7}$.

Anche in altri sensi Nietzsche si presenta come filosofo particolarmente cinematografico: infatti il pathos, l'emozione e il sentimento assumono nei

6 NIETZSCHE, F. Der Wille zur Macht, edizione del 1911, a cura di Otto Weiss, 481; trad. it. La volontà di potenza, a cura di Maurizio Ferraris e Pietro Kobau, Bompiani, Milano, 2005. Per approfondire il prospettivismo attraverso la letteratura secondaria rimando soprattutto alle opere di J. Derrida e G. Deleuze in Francia e di A. Nehamas e A. Danto in America.

7 Si vedano ad esempio, MAGNUS, B. The Deification of the Common Place: Twilight of the Idols. In: SOLOMON, R. C.; HIGGINS, K. M. (Ed.). Reading Nietzsche, New York, Oxford University Press,1988. p. 152. 
testi di Nietzsche, come nel cinema, un ruolo centrale. L'ambiguità e la messa in discussione di tutto quanto prende in considerazione si ritrova nel cinema non più che nel filosofo: egli infatti critica radicalmente la ragione, la verità e la morale rigidamente fissate. L'allusione al mondo attraverso simboli e metafore accomuna il carattere inevitabilmente metaforico del cinema alle pagine del filosofo tedesco, soprattutto in Su verità e menzogna. Inoltre, svanita l'illusione del realismo cinematografico, ci si rende conto che in molti film il confine tra realtà e sogno è davvero labile e la metaforicità diventa onirica: proprio Nietzsche, in La nascita della tragedia, sostiene con forza una concezione dell'arte legata al sogno e alla sua interpretazione.

\subsection{Registi nietzscheani: Orson Welles e Peter Greenaway}

Alcuni registi hanno caratteristiche che, più o meno consapevolmente, rievocano il pensiero del filosofo tedesco.

Per esempio i film di Orson Welles sembrano passare in rassegna $i$ punti principali della critica della verità del filosofo di Röcken ${ }^{8}$ : il mondo vero non esiste e, se esistesse, sarebbe inaccessibile, inevocabile e, anche se fosse evocabile, sarebbe inutile superfluo. Anche Welles non ha mai smesso di combattere il sistema del giudizio: come dice Deleuze, non esiste valore superiore alla vita, la vita non deve essere giudicata né giustificata, è innocente, possiede "l'innocenza del divenire", al di là del bene e del male".

Nei suoi film Peter Greenaway sembra aver esteticamente fatto proprio il precetto esistenziale dell'eterno ritorno: non c'è infatti un solo momento che non sia reso il più interessante ed essenziale possibile. Ogni immagine è come un dipinto, un istante che va vissuto nella sua pienezza. In alcuni suoi film mostra come anche storie apparentemente non interessanti possano essere rese pregne se vissute con l'intensità che meritano i momenti eterni. Inoltre, anche se la comprensione da parte dello spettatore risulta lacunosa, $\mathrm{i}$ dipinti-sequenza di questo regista sono individualmente così belli da renderli interessanti e meritevoli di essere vissuti a prescindere dal resto della storia. In altri film, al contrario, i momenti e le immagini, per quanto apparentemente

8 Per la precisione del primo Nietzsche, cioè fino alla Gaia scienza.

9 DELEUZE, G. Cinema 2. L'image-temps, Paris: Minuit, 1985. tr. it. Cinema 2. L'immagine-tempo, Ubulibri: Milano, 2004. p. 154-155. 
marginali e trascurabili (per esempio un insetto al margine della scena), sono resi estremamente importanti per il loro significato riguardo a tutto il resto della narrazione anche a prescindere dalle abituali catene causali. Infine tutti i suoi film cercano di dire "si" a tutto quanto la realtà può metterci di fronte, proprio come suggerisce Zarathustra: ecco allora la proposta di accostamenti anche stridenti tra orrore, sesso, amore, ecc.

\subsection{Film: I magnifici sette (1960, Regia Di John Sturges)}

Per brevità preferisco non soffermarmi sulla trama di questa e delle successive opere prese in considerazione: si tratta comunque di opere assai celebri.

Questo celebre western si presta ad illustrare alcune delle tematiche dell'interpretazione più diffusa, anche se non sempre corretta, del pensiero di Nietzsche. Innanzitutto c'è l'eroe, anzi gli eroi. Infatti i pistoleri del film sono coraggiosi, temerari, sicuri di sé, senza famiglia, senza legami tradizionali né con persone né con luoghi: ricordano quindi certi tratti superficiali dell'Übermensch. Inoltre questi pistoleri accettano il loro incarico gratuitamente e senza gloria, attirati dal rischio e dall'opportunità di mettersi alla prova. Una generosità quindi che non sorge dai valori morali cristiani o dalla solidarietà, che Nietzsche critica in quanto morale dei deboli, ma espressione di vitalità e di inconsapevole volontà di potenza. Questi pistoleri dispensano la stessa violenza dei cattivi che combattono: ciò che li differenzia non è la loro bontà ma la gratuità e l'assoluta mancanza di calcolo e di interesse che li contraddistingue. Ciò è ben rappresentato dalla scena in cui, alla fine del film, il capo dei cattivi, meschino e calcolatore, muore chiedendo la ragione di questo loro sacrificio al capo dei pistoleri. Ovviamente non riceve risposta perché non c'è risposta: le ragioni e la causalità delle azioni non trovano qui maggiore ospitalità che nel pensiero del filosofo tedesco.

Nello stesso film però si ritrovano anche i valori che Nietzsche rifiuta in quanto legati ad interessi fossilizzati, ad una morale imposta falsamente. Il fatto interessante è che a proporli è la parte cosciente e consapevole degli eroi, che, rispetto alla loro istintiva pulsione eroica gratuita, sembra avere sensi di colpa cristiani o comunque moralistici. Rimpiangono quindi la mancanza di una famiglia, di una dimora fissa, deprecano la loro violenza e giudicano la vita dei contadini assai più eroica. Questo moralismo 
risulta posticcio nel contesto del film: dopotutto sono stati i pistoleri con le loro armi e con la loro generosità non cristiana a salvare il villaggio. Il film sembra quindi rimanere indietro rispetto alla spregiudicatezza e alla a-moralità dal filosofo: l'emozione, il sentimento e l'istinto sembrano meno rilevanti nella impostazione data dagli autori al film in quanto essa mostra attaccamento alla morale tradizionale, all'essere del contadino rispetto al divenire e al cambiamento dei pistoleri, che in effetti vengono paragonati al vento che passa e va.

\subsection{Film: Arancia meccanica (1971, Regia Di Stanley Kubrick)}

Prima di affrontare questo film è necessaria una breve premessa in due punti.

Nietzsche in alcune pagine descrive la società come una prigione, dove l'uomo, dimentico di se stesso e della propria natura, è rinchiuso da sbarre fatte di valori falsi e opprimenti, di ideali sterili e anonimi. La società altro non è che l'omologazione dell'individuo, il tentativo di limitare l'animo umano, libero e creativo per natura, tramite le false illusioni della ragione. L'uomo è perciò uno schiavo e ad esercitare il controllo su di esso sono le grandi ipocrisie della morale, come le religioni. Una delle ultime opere di Nietzsche, Der Antichrist, ne è il fulgido esempio: essa riconosce il cristianesimo come una tecnica di controllo e annientamento della vita. Strumentalizzando la paura della morte, il cristianesimo impone la repressione degli istinti, costringendo al senso di colpa, all'angoscia e alla sofferenza in nome di una falsa promessa (il paradiso). Vede quindi una società decadente, dove la morale è solo uno strumento di vendetta dei deboli sui forti.

Nietzsche sostiene che la massima espressione della civiltà ellenica si sia avuta con l'avvento della tragedia: la capacità tragica di mettere a nudo la natura umana, ossia il connubio tra le due grandi forze che animano $i$ greci, l'apollineo e il dionisiaco, dischiude la comprensione della realtà e dell'essere umano stesso. Con Socrate, questo dualismo viene a mancare, in quanto egli impone il primato della ragione sull'irrazionalità, escludendo l'elemento dionisiaco: un dominio che risulta però fittizio. Se la tragedia greca è morta, non è morta e mai morirà la dimensione tragica dell'uomo, che si sfoga nel malessere della società. Tale malessere è rappresentato da Kubrick in Arancia Meccanica. E' possibile per un uomo vivere in modo 
autentico, libero dalle illusioni? Come si pone l'uomo nei confronti della società e dei propri simili? Nietzsche ritiene che, se liberato dal giogo della religione e della morale, dai vincoli della ragione, l'uomo può diventare spirito libero e liberare la propria parte irrazionale abbandonandosi alla danza dionisiaca del suo istinto.

In Arancia Meccanica, il protagonista, Alex, è lo spirito libero: non ha paura di nulla e si abbandona con coraggio all'irrazionalità. E' la manifestazione, caotica e frenetica, di tutti gli istinti e gli impulsi di cui la società ci ha privato, è la natura umana messa a nudo, libera, svincolata dagli schemi della ragione; è la vita, pura e semplice. Nell'esercizio della sua amata "ultraviolenza", egli è felice come un bambino. Per quanto possa compiere nefandezze e crudeltà, suscitando la disapprovazione dello spettatore, quest'ultimo non riesce a non rimanerne affascinato. Alex possiede vitalità, coraggio ed intelligenza, ma non si può non vedere che è completamente malvagio. Nello stesso tempo c'è uno strano tipo di identificazione psicologica dello spettatore, per quanto il suo comportamento possa respingerci: è sempre assolutamente onesto nel suo racconto in prima persona, fino al punto di soffrirne, ed inoltre, ad un livello inconscio, forse tutti abbiamo in comune certi aspetti della sua personalità. Infatti gli uomini sono tutti spiriti liberi in potenza ma la stragrande maggioranza di essi lascia "vincolare" la propria spontanea volontà di potenza. Questa non va però confusa con il predominio e il potere: la volontà di potenza non è semplice affermazione sugli altri, come quella che esercitano Pete, Dim e Georgie (gli scagnozzi di Alex); non è mediocre e volgare violenza. E' piuttosto la volontà di affermare se stessi e la propria natura che, purtroppo, scaturisce in violenza.

La volontà di potenza significa anche affermare la propria prospettiva sul mondo. Però, nel film, la prospettiva del protagonista non riesce ad affermarsi: mentre i suoi scagnozzi rimangono fedeli al loro vile opportunismo, la società rifiuta Alex e dopo aver fallito la "rieducazione" decide di servirsene per controllare gli altri (la riconciliazione finale con il Ministro della Giustizia, che in ospedale lo imbocca con il cucchiaio in una scena carica di allegoria). La sua volontà di potenza viene castrata, combattuta dalla società e perciò egli non riesce ad andare oltre lo stadio di spirito libero, ad essere Ubermensch. 
Anche nell'elemento musicale del film si può cogliere un nesso con Nietzsche. Alex ha una grande passione per Beethoven ${ }^{10}$ per la sua musica feroce e passionale, elementi che lo avvicinano a Wagner, il musicista ammirato, fino alla rottura, da Nietzsche. Come sosteneva Wagner, la loro musica cerca l'inesprimibile, l'irrazionale, riflettendo la vita elementare dei sensi e rigettando l'uomo nella sua originaria dimensione.

\subsection{Film: Gli Spietati (1992, Regia Di Clint Eastwood)}

Sebbene si tratti di un western come I magnifici sette, in questo film domina un'ideologia assai diversa da quella moralista. Il protagonista, Bill Munny, è un poco di buono, un pistolero assassino specializzato: è solo la controfigura del cowboy coraggioso e giustiziere. Sembra dover pagare un debito morale nei confronti dell'universo femminile ma soprattutto pare confrontarsi con un conflitto interno: vuol mostrare di essere ancora in grado di fare quello che faceva prima che la defunta moglie lo costringesse ad una tranquilla vita contadina, cioè il killer spietato.

Anche qui si trova la contrapposizione tra la vita tranquilla, familiare e morale da una parte e la vita rischiosa ed effimera del pistolero dall'altra. In questo caso però l'alfiere della vita calma e morale non sono i contadini ma lo sceriffo Little Big Daggett: si tratta però palesemente di una morale dei mediocri, di violenza dei deboli. Con la scusa di pacificare il paesino di Big Whiskey lo sceriffo vuole imporre la propria giustizia, che però ignora qualsiasi valore della persona e viene somministrata solo attraverso accordi e considerazioni burocratiche e materiali. Munny attacca questa labile costruzione di morale ipocrita uccidendo lo sceriffo ed i suoi aiutanti, colpevoli di aver cercato di trasformare la loro volontà di potenza in rigida morale collettiva: l'eroe, anche se stanco e invecchiato, riesce a sconfiggere il moralista, la morale del gregge.

La violenza è presentata come elemento ineliminabile. Munny pensa di potersi ritirare ma in realtà alla prima occasione torna di buon grado alla vita violenta. Lo sceriffo maschera la propria violenza e prepotenza dietro l'ipocrita pretesa di imporre la giustizia: egli in un certo senso rappresenta

\footnotetext{
${ }^{10}$ Questo sembra essere un tratto piuttosto comune tra i personaggi cinematografici che vivono "sopra le righe", come ad esempio il poliziotto folle del film Leon.
} 
la violenza delle istituzioni, dello Stato. Esiste però una differenza: il primo, nonostante la sua violenza, mostra una completa assenza di cattiva coscienza moralistica; lo sceriffo invece è moralista, debole e vigliacco. Viene proposta un'immagine della vita necessariamente e inevitabilmente violenta e opportunistica: astenersi dalle offese, dalla violenza rischia di diventare una negazione della vita, un principio di dissoluzione e di decadenza in quanto imposto dalla volontà di potenza di qualcuno.

Due concettimmagine di questo film sono piuttosto evidenti: la violenza e l'eroismo. Più nascosto è il rapporto dell'intellettuale con la violenza: quest'ultimo, rappresentato dal biografo opportunista W.W. Beauchamp che cambia padrone con maggiore frequenza di Arlecchino, è particolarmente interessante in quanto mostra come l'ipocrisia degli intellettuali sia talvolta complice della violenza da cui pretendono di affrancarsi.

\subsection{Film: Assassini nati (1994, Regia Di Oliver Stone)}

Ecco un film realmente a-morale: difficilmente Nietzsche avrebbe potuto accusarlo di moralismo o di fondazionalismo epistemologico. A differenza di quanto avviene in altri qui non si distingue tra violenza giusta perché buona, controllabile e razionale, e violenza cattiva, perché ingiusta e irrazionale: la violenza per Nietzsche caratterizza l'uomo e non ammette di essere sottoposta ai dualismi giusto-ingiusto.

La violenza è mostrata in tutta la sua inevitabile naturalezza ed esuberanza senza motivazioni o giustificazioni di ordine morale, psicologico o sociologico: si tratta di assassini nati ${ }^{11}$. Uno degli stessi protagonisti, interrogato dalla polizia, afferma che lui uccide né più né meno come fanno tutti gli altri esseri viventi: è nella loro natura. Tale inevitabilità è ribadita nel racconto allegorico che fa un indiano di una donna uccisa da un serpente che ella aveva aiutato (simile alla spesso citata fiaba della rana e dello scorpione). Tali fiabe trovano nel film la loro realizzazione sia nel caso dello stesso indiano, sia nel caso di un presentatore televisivo che prende le parti dei due assassini ma che essi, loro malgrado si vedono costretti ad uccidere senza motivo al di là della loro natura: "mi mancherà", si trovano a dire, senza alcuna ombra di ironia, contemplando il suo cadavere.

${ }^{11}$ Il titolo originale Natural Born Killers contiene appunto il termine "natural". 
Il film suggerisce che la natura escluda del tutto la moralità, pur senza né rifiutarla né metterla in discussione: c'è solo essere, non dover essere. Anche volendo spiegare l'origine della violenza dei due protagonisti nei conflitti a cui hanno assistito in ambito sociale e familiare, si sposta solo a lato il solito scontro tra diverse violenze, tra diverse forme di vita, tra diverse volontà di potenza: non c'è quindi posto per valutazioni di tipo morale.

I due criminali sono nature semplici che possono essere viste come incarnazione dell'uomo in salute fisica e spirituale senza rimorsi e complessi di colpa. Si contrappongono alla società dei deboli, che li tratta da delinquenti ma esercita a sua volta la violenza attraverso le istituzioni riconosciute. Anche i personaggi che rappresentano le istituzioni sono infatti alla fine artefici di violenza, anche se di diverso tipo, cioè socializzata e moralizzata: come tali vengono messi in discussione nel film (la rivolta nel carcere, la critica dei media). Sembra che il delinquente invece sia un uomo forte in condizioni sfavorevoli: gli manca l'ambiente selvaggio, un'esistenza più libera e pericolosa. Purtroppo la frustrazione dei divieti imposti e la conseguente necessità di agire di nascosto $\mathrm{e}$ in modo subdolo lo portano a degenerare.

Tutte le tecniche caratteristiche del cinema concorrono a sottolineare la pervasività della violenza: le deformazioni, gli stacchi, il sonoro, le inquadrature, la rapidità delle immagini. Vengono inoltre sovrapposte metaforiche sequenze di violenza tra animali (da insetti a grandi mammiferi). Anche il multiprospettivismo concorre a creare questa atmosfera di violenza: sembrano infatti esserci diversi narratori che parlano tutti assieme, accomunati solo da una palese freneticità e alterazione mentale.

\section{APPENDICE 1}

Al fine di mostrare come il cinema possa non solo essere analizzato filosoficamente ma anche utilizzato a scopo didattico all'interno di corsi di filosofia propongo in questa e nella successiva appendice due schede esemplificative. 


\section{Proposta di utilizzo didattico-filosofico del film Angel's Dance (di David L. Corley)}

Guida per la visione e la discussione del film Professione Killer (tit. orig. Angel's Dance) come parte delle attività usate per proporre elementi e conseguenze della filosofia di Nietzsche in un corso introduttivo.

\section{Introduzione}

E'possibile usare l'etica di Nietzsche per giustificare le azioni di un assassino? Il film Professione Killer, in parte serio in parte scherzoso, presenta una tale situazione. Anche se da una parte esso può essere considerato una parodia della vulgata delle idee di Nietzsche e del buddismo zen, dall'altra fornisce un esempio di una possibile interpretazione delle conseguenze etiche della filosofia di Nietzsche. La visione e la discussione del film è un'opportunità per gli studenti di divenire maggiormente consapevoli del pensiero del filosofo tedesco e delle sue conseguenze. Si tratterebbe di un modulo da inserire in un corso introduttivo all'etica in cui siano presenti anche approcci tradizionali, per esempio, a Aristotele, Kant, Mill e Nietzsche stesso.

\section{Cose da sapere}

Trama del film (preventivamente per il docente)

Il capo di una banda mafiosa di Chicago, avendo bisogno di nuovi sicari, affida un potenziale aspirante (Tony Greco) all'addestramento di un assassino di grande esperienza (Steve Rosellini). Rosellini vive in una casa in stile giapponese sulla spiaggia (California), crede nella reincarnazione, propina massime Zen, mangia vegetariano, è ambientalista, ammira e cita Nietzsche. Il manuale che fornisce all'allievo è Al di là del bene e del male; inoltre durante il film i due killer armeggiano anche con The Portable Nietzsche (la celebre traduzione in Inglese delle opere di Nietzsche edita da W. Kaufmann). Una parte dell'addestramento di Tony consiste nell'uccidere una vittima innocente estratta a caso dall'elenco del telefono: Angelica (Angel) Chaste, una donna a dir poco strana, intelligente, piena di risorse e anch'essa frequentatrice delle opere di Nietzsche. La situazione si complica quando, dopo un primo tentativo di omicidio fallito, la donna reagisce in 
modo inaspettato tramutandosi da preda a predatrice: cambia aspetto, si arma e diventa aggressiva e pericolosa. I ruoli si invertono completamente: mentre Tony perde sempre più le sue motivazioni, Angel assume perfettamente il ruolo del killer professionista. Tony si innamora della sua ex-vittima mentre lei diviene il nuovo allievo del maestro Rosellini. Il film si conclude con Angel e Rossellini che sfrecciano in una macchina sportiva e la musica tratta da Zorba il Greco.

Background filosofico

Gli studenti devono avere una precedente conoscenza di alcune nozioni base della filosofia di Nietzsche, come la volontà di potenza, il nichilismo, la critica della cultura europea, l'Ubermensch e l'eterno ritorno. La scelta dei testi a questo scopo è lasciata al docente.

\section{Come si procede}

- Preparare una lista di domande (sotto ho riportato un esempio)

- Proiezione del film agli studenti

- Far prendere in considerazione le domande (già preparate) durante la visione del film e farne preparare altre

- Rivedere alcuni spezzoni del film (sotto ho riportato un esempio)

- Discutere in gruppi le domande iniziali, le altre sorte dopo ed aspetti del pensiero di Nietzsche che sono emersi dal film

- Far scrivere un paio di cartelle sulla relazione tra il film e la filosofia di Nietzsche

\section{Domande iniziali (esempio)}

1. Come sono incorporati nel film elementi della filosofia di Nietzsche?

2. Chi nel film può essere identificato con l'Ubermensch?

3. Qual è l'evoluzione di Angel e Tony durante il film?

4. Come è presentata la pretesa di Rossellini di essere etico? Come giustifica egli tale dichiarazione? Discutete a favore e contro tale dichiarazione. In che rapporto è tale dichiarazione con la filosofia di Nietzsche?

5. Qual è, se esiste, la relazione tra la filosofia di Nietzsche e il buddismo zen?

\section{Spezzoni/dialoghi del film (esempio)}


1. Scena in cui Rosellini consegna a Tony come manuale il testo $\mathrm{Al}$ di là del bene e del male.

2. Dialogo

Tony [legge]: "Pochi sono fatti per l'indipendenza, è un privilegio dei forti”.

Rosellini: "La tua più grande difficoltà è non permettere che ti sia imposta la morale della società".

Tony: "Sei un killer; tu non hai una morale".

Rosellini: "Sono uno dei pochi moralisti che incontrerai mai".

Tony: "Oh, il killer morale!"”

Rosellini: "Ho una morale perché ho un'etica: un codice di comportamento professionale".

3. Dialogo (da Professione Killer (tit. orig. Angel's Dance))

Rosellini: "Io credo nella reincarnazione. La morte non è la fine... è la parte di un viaggio, del cammino lungo la vita. Il tipo di persone che uccido, insomma...non raggiungerebbe comunque l'illuminazione in questa vita; così in un certo senso io le aiuto a procedere verso la prossima vita".'

4. Scena in cui Angel e Rosellini si scambiano citazioni di Nietzsche.

\section{APPENDICE 2}

\section{Bozza di proposta di utilizzo didattico-filosofico di una sequenza del film Mon Oncle d'Amerique (di Alain Resnais)}

Sequenza: La sequenza finale del film Mio zio d'America si presta in modo particolare a descrivere il prospettivismo di Nietzsche. Qui propongo solo uno spunto di riflessione su cui eventualmente impostare un'attività didattica.

La sequenza finale del film apre con ciò che sembra un paesaggio di campagna. Il campo s'allarga e lo spettatore si accorge che tale paesaggio si trova in realtà nel bel mezzo di una città; gradualmente si rende conto che si tratta di un affresco iperrealista dipinto sul lato di un edificio: i suoi dettagli sono perfetti, le linee nette, la superficie liscia e omogenea. L'obiettivo si avvicina alla parete e ci rende conto della presenza dei mattoni, della calce tra essi, delle increspature della superficie, della irregolarità dei colori; il 
paesaggio si perde sostituito da confuse pennellate. Dall'opera iperrealista si passa ad un'opera impressionista o addirittura espressionista. Il film chiede: qual è il vero dipinto?

Riflessione: Non si possono vedere allo stesso tempo due versioni del dipinto o due gruppi di linee. Il dipinto è tutte queste versioni sommate insieme, però questa peculiare somma non produce un singolo oggetto reale che si contrappone all'apparenza delle varie versioni. Il dipinto è solo queste apparenze, ognuna presa da un particolare punto di vista. Quindi non si tratta di apparenze di una realtà e noi non abbiamo alcun diritto di parlare di apparenze.

Collegamento a Nietzsche: Il prospettivismo di Nietzsche sostiene che non esistano fatti ma solo interpretazioni. Non esiste la realtà contrapposta all'apparenza in quanto ogni conoscenza è inevitabilmente prospettica: essa "semplifica" e riduce i "dati" (termine molto delicato) possibili in base al nostro punto di vista, ai nostri bisogni.

Data de registro: $22 / 12 / 2013$

Data de aceite: 19/11/2014 